\title{
New Friends: Social Robots in Therapy and Education
}

\author{
Marcel Heerink $^{1}$ - Bram Vanderborght ${ }^{2} \cdot$ Joost Broekens $^{3} \cdot$ Jordi Albó-Canals $^{4}$
}

Published online: 25 August 2016

(C) Springer Science+Business Media Dordrecht 2016

This special issue reflects this multidisciplinary field of social robots in therapy and education with a broad collection of contributions, concerning technical, therapeutic, educational and ethical issues. The application of social robots in therapy and education is an emerging field. This development is fueled by the fact that these 'new friends' become more sophisticated, available and affordable. In recent years there has been an enormous increase of projects in which social robots are used successfully for groups with special needs, such as people with dementia, hospitalized children and children with autism. These projects demand expertise from a wide range of disciplines, like psychology, nursing, occupational therapy, physiotherapy, AI, robotics and education to meet the technical opportunities with the development of therapeutic and educational practice.

The first five papers are addressing usage and relevant methodological issues concerning the use in therapy or edu-

Marcel Heerink

m.heerink@windesheimflevoland.nl

Bram Vanderborght

bram.vanderborght@vub.ac.be

Joost Broekens

joost.broekens@gmail.com

Jordi Albó-Canals

jalbo@salleurl.edu

1 Robotics Research Group, Windesheim University, Almere, The Netherlands

2 Brussels Human Centered Robotic research center (BruBotics), Vrije Universiteit Brussel, Brussels, Belgium

3 Intelligent Systems, Delft University of Technology, Delft, The Netherlands

4 Engineering School, La Salle - Ramon Llull University, Barcelona, Spain cation of either a specific social robot, a specific category of social robots or social robots in general.

A first well-known application field of socially assistive robots is autism. Huijnen et al. studied the potential added value of therapy robot Kaspar to the therapy or education goals for children with an autism spectrum disorder (ASD). By conducting focus group sessions and an online questionnaire the expectations of practitioners were investigated in domains such as communication, social/interpersonal interaction and relations, play and preschool skills.

Another challenge related to usage in real life is the acceptance of personal robots in the home. Jochum et al. described an innovative approach to influence the acceptance of care robots using theatrical performance. They investigated how audiences perceive social robots interacting with humans in a future care scenario through a scripted performance.

In the subsequent contribution, Özcan et al. investigate the issue of suitable robot forms for particular therapeutic domains. They present a novel concept of interactive devices, called 'transitional wearable companions': soft interactive animal shaped devices, able to evoke emotions and giving a continuous reassuring physical contact. Their application is to support therapy and foster social skill development in children with autism spectrum disorder (ASD), giving a therapist a range of possibilities with in- and output control, and gather biosensor information on the child's physiological and emotional state.

A contribution by Looije et al. reports on a study of how social robots can be used to support the development of selfmanagement of children with diabetes. They develop profiles for further personalization of such a robot, and describe general requirements for mediating the support of parents and caregivers. 
Finally, a study by Scholten et al. addresses the application of hygiene protocols in a clinical environment and establishes to what extend such protocols can be applied to robotic animals that are currently used in this setting. Their paper features essential principles to be observed by researchers in this field and presents a framework for further research.

The next two contributions address design issues of socially interactive robots to be applied in different kinds of therapy. Issues include affect and embodiment.

De Beir et al. address the design of affective expressive capabilities of social robots in the form of eyebrows on a NAO robot (that does not have eyebrows by default). They present a technically clever solution to add eyebrows as a module to the NAO and show in a user study that the expressions of sadness versus happiness are reliably recognized even when the robot performs gestures at the same time (they test two different gestures).

Canamero et al. present the design of a cognitively and motivationally autonomous affective robot toddler. It has been developed to support perceived self-efficacy and emotional wellbeing in children with diabetes, applying an 'Embodied AI' approach, which is argued to fulfill the therapeutic and affective requirements of the target group beyond other approaches commonly used in assistive robotics and child-robot interaction.

Finally, five papers focus on the technical, ethical and societal landscape needed for therapeutic and educational use of social robots.

Reppou et al. propose a software architecture that facilitates deployment and flexible addition of functionality to smart home systems, including robots. They propose a cloudbased solution that facilitates the development of novel apps, together with offline machine learning modules as well as databases that learn and store user data. Of course they pay extra attention to data privacy, for example, in the way Robot apps are deployed in the system.

A study by Zubrycki et al. presents a project named Roboterapia. This project concerns an environment supporting the needs of therapists working with children with special developmental needs, developed and tested in the field of autism treatment. The authors report on the developmental process, eliciting and specifying the needs of therapists and the produced prototype devices that could support different aspects of therapists' work.
Sedenberg et al. address the important topic of ethics in deploying robots in healthcare and in the home environment. Using a series of hypothetical case they build a strong argument for the necessity of ethical guidelines, particularly needed in a response to the data gathering potential of such robots when used in a home or care setting.

In a contribution by Ienca et al. ethical recommendations are discussed for research and practice concerning social and assistive robotics applied in dementia care to prevent slow adoption, incorrect implementation and inappropriate use. It delineates the ethical landscape and provides recommendations for design and use aimed at protecting users and maximizing the benefit in assisting this vulnerable population.

The final paper of this issue by Maartje de Graaf addresses the ethics of human-robot relationships. The author explores the nature of this new genre of social interaction and argues that we have to view its development as an evolution of a new species, becoming 'a new form of living glue between our physical world and the digital universe...'. She establishes the implications this has on future generations, issues that should be addressed in further research and questions that could guide a discussion on the ethical considerations of human-robot relationships.

Overlooking these contributions, we see academic research in social robotics moving closer to practice in therapy and education. This obviously stresses the importance of involving health care professionals in this research understand their needs and concerns, but it also convinces us that ethical considerations as well as political and legal issues can be expected to become more and more pressing with the narrowing of the gap between research and its application in this field.

Acknowledgments We wish to express our gratitude to Vanessa Evers and Shuzhi Sam Ge for their confidence and cooperation, to Martina Heinemann for her editorial assistance and to all the reviewers for their support in maintaining the high standard of the journal.

Guest editors, Marcel Heerink Bram Vanderborght Joost Broekens Jordi Albó-Canals 